\title{
Escherichia coli strain 0111
}

National Cancer Institute

\section{Source}

National Cancer Institute. Escherichia colistrain 0111. NCI Thesaurus. Code C86881.

A species of facultatively anaerobic, gram negative, straight rod shaped bacteria assigned to the phylum Proteobacteria. This shiga-toxin producing species is chemoorganotrophic, capable of both respiratory and fermentative metabolism. It is the most common non-0157 E. coli strain that is the causative agent of bloody diarrhea and hemolytic uremic syndrome in the United States. Escherichia coli and strain 0111 can be isolated from livestock such as cattle. 